Sādhanā Vol. 40, Part 3, May 2015, pp. 985-1000. (C) Indian Academy of Sciences

\title{
Role of viscoelasticity in instability in plane shear flow over a deformable solid
}

\author{
PARESH CHOKSHI \\ Indian Institute of Technology, New Delhi 110 016, India \\ e-mail: paresh@chemical.iitd.ac.in
}

MS received 18 July 2014; revised 5 December 2014; accepted 9 January 2015

\begin{abstract}
The stability of the flow of a viscoelastic fluid over a deformable elastic solid medium is reviewed focusing on the role played by the fluid elasticity on the earlier known instability modes for the Newtonian fluids. In particular, two classes of modes are emphasized: the viscous mode for the creeping flow, and the wall mode for high Reynolds number flow. The flow geometry is restricted to plane Couette flow of fluid supported on elastic substrate of finite thickness. The viscoelastic fluid is described using the Oldroyd-B model and the dynamics of the deformable solid continuum is described by either Hookean or neo-Hookean elastic model. In the limit of $R e \rightarrow 0$, the introduction of fluid elasticity delays the onset of instability and for sufficiently viscoelastic fluid with dilute polymer concentration, the instability is suppressed rendering the flow stable. For concentrated solution and polymer melt, the instability persists, but with higher value of critical shear rate than for the Newtonian fluid, indicating stabilizing role of fluid elasticity in creeping flow regime. However, for high Reynolds number flow of dilute polymer solution, the polymer addition plays a destabilizing role for wall modes, indicated by reduction in critical Reynolds number by an order of magnitude.
\end{abstract}

Keywords. Hydrodynamic stability; flexible surface; flow transition; viscoelasticity.

\section{Introduction}

The flow over deformable substrates has been an area of active research over couple of decades due to rich complexities generated by the elasto-hydrodynamic coupling. The delay of turbulence in boundary layer flow over deformable surfaces as found by pioneering experiments of Kramer (1957) has created a strong interest in materials coated with elastic solid media. For bounded flow, like flow through gel-walled tube, the experiments by Krindel \& Silberberg (1979) showed the opposite role of wall deformability in flow transition. Here, the transition Reynolds number was found to be much smaller than 2100, the corresponding value for the rigid tubes. The early instability is attributed to the growth of wall displacement fluctuations caused by shear deformation of wall material due to flow. Motivated by this finding, extensive studies of fluid flow in tubes and channels bounded by flexible walls have been carried out (Kumaran et al 1994; Kumaran 1995, 1996, 1998a, b; Shankar \& Kumaran 2001, 2002; Gkanis \& Kumar 2003; 
Eggert \& Kumar 2004; Kumar \& Shankar 2005). The results of these studies indicated that there are at least three modes of instability in flexible-walled tubes and channels which are qualitatively different from those in rigid tubes and channels, namely viscous modes, wall modes and inviscid modes, depending upon the regime of flow operation. Thus, the gel-coated substrates can be considered as potential candidates to generate instabilities. An excellent review and classification of these instabilities has been covered by Kumaran (2000).

Recently, a renewed interest in the flow through deformable tubes and channels experiments has been generated by experiments of Verma \& Kumaran $(2012,2013)$ for a flow through flexible tube. It has been shown that the flow undergoes transition from parabolic laminar profile to complex profile at Reynolds number as small as 500, suggesting a strong influence of flexible wall on flow transition. Further, the development of microfluidic devices in recent time has further revived the interest in soft substrates. The flow instability even in the absence of inertia can be exploited to gain manifold enhancement of the mixing and other transport properties in low Reynolds number flows.

In one of the first analyses of bounded flow, Kumaran et al (1994) performed the linear stability of a plane Couette flow past a flexible surface in the low Reynolds number regime. The deformable wall was modeled as an incompressible linear viscoelastic solid. The authors observed that the elasto-hydrodynamic coupling between the fluid flow and the wall dynamics renders the flow unstable when the imposed dimensionless shear rate, $\Gamma=V \eta /(G R)$, exceeds a certain critical value $\Gamma_{c}$. Here, $V$ is top-plate velocity, $R$ is channel width, $\eta$ is fluid viscosity and $G$ is the shear modulus of the elastic solid underneath the flow. The instability referred to as the viscous instability is driven by a discontinuity in the strain rate across the fluid-solid interface. The destabilizing mechanism is proposed to be the transfer of energy from the mean flow to the fluctuations due to the shear work done by the mean flow at the interface.

For low Reynolds number flow, the shear strain the elastic solid is of the order of unity. Hence, the classical linearized model for solid elasticity becomes inappropriate and it is imperative to incorporate the finite strain terms in the constitutive relation for the elastic solid. This argument led Gkanis \& Kumar (2003) to analyze the viscous instability using the more appropriate neo-Hookean elastic model for the flexible surface. Unlike the Hookean model, the nonlinear neo-Hookean model exhibits non-zero normal stress difference which results in a shortwave instability mode which was absent in the earlier analysis of Kumaran et al (1994). The neoHookean model predicted lower value of $\Gamma_{c}$, the critical shear rate for the onset of instability. The viscous instability for plane Couette flow has been confirmed by the experiments of Kumaran \& Muralikrishnan (2000), Muralikrishnan \& Kumaran (2002) and Eggert \& Kumar (2004). In the experiment, the elastic solid consists of polyacrylamide gel of thickness around $4.5 \mathrm{~mm}$, which supports a viscous fluid layer of thickness around $300 \mu \mathrm{m}$ to $1000 \mu \mathrm{m}$ in a parallel-plate rheometer. The onset of instability is identified by a sharp increase in the apparent viscosity (assuming laminar flow) when the imposed shear rate exceeds a certain critical value. The experimental value of critical shear rate required for the on-set of instability was found to be in good agreement with the theoretical predictions of Kumaran et al (1994) for a wide range of gel thicknesses and elastic moduli.

In the other extreme limit of very high Reynolds number, the flow is also found to be unstable to wall modes. For this class of modes, the disturbance vorticity in the fluid is confined to a thin region near the fluid-solid interface of thickness $O(R e)^{-1 / 3}$ smaller than the channel width $R$. The corresponding wall modes for the rigid surface case are known to be always stable (Corcos \& Sellars 1959; Gill 1965a, b). However, for flow past a deformable surface, there exists a series of unstable wall modes indicating the existence of critical Reynolds number $R e_{c}$ for the onset of instability (Shankar \& Kumaran 2001, 2002). The numerical and asymptotic analysis suggests 
the scaling of $R e_{c} \sim \Sigma^{3 / 4}$, where $\Sigma=\rho G R^{2} / \eta^{2}$, dimensionless parameter characterizing the fluid-solid system.

While most of the attention is restricted to the flow of Newtonian fluids, real fluids in practical applications are viscoelastic. Few studies have been carried out to examine the role of fluid elasticity on the known modes of instabilities and any potentially new modes introduced by the non-zero normal stress difference in the viscoelastic fluid. For the viscoelastic fluid, considerable amount of work has been carried out to study the stability of a shear flow past a rigid surface and to investigate the nature of eigen-spectrum for the growth rate (Gorodtsov \& Leonov 1967; Renardy 1992; Wilson et al 1999). In most studies, the viscoelastic fluid is described either by the upper convected Maxwell (UCM) model or the Oldroyd-B model, wherein the polymer chains are treated as the elastic dumbbells. For the flexible surface, Shankar \& Kumar (2004) analyzed the creeping flow of UCM fluid over linearly viscoelastic solid. The analysis predicts an unstable viscous mode when a parameter $\Gamma=V \eta /(G R)$ exceeds a certain critical value. The analysis shows that the fluid elasticity has a strong stabilizing influence on the unstable viscous mode for the Newtonian fluid. Chokshi \& Kumaran (2007b) analyzed the linear stability of an Oldroyd-B fluid flow past a linearly viscoelastic solid. Later, analysis was carried out using the neo-Hookean elastic solid (Chokshi \& Kumaran 2007a), which is reviewed in some detail in this paper.

We review the linear stability analyses to examine the role of fluid elasticity on the instability modes excited by wall deformability. Here, we emphasize two classes of instability modes: the instability in creeping flow regime (in the absence of flow inertia), and the wall mode instability for moderate to high Reynolds number flow regime.

\section{Creeping flow regime}

In the absence of inertia, the Newtonian fluid flow over a flat rigid surface is always stable, as the viscous forces dissipate any disturbance energy leading to time-independent laminar flow in instantaneous manner. Also, the absence of nonlinearities ensures no means of transfer of energy from the base-state to the disturbance. Rendering the surface deformable, however, introduces nonlinearities and thus introducing instability even in the absence of inertia (Kumaran et al 1994). This mode is referred to as the viscous instability mode. Now, if one considers a viscoelastic fluid, the time-dependence, due to non-zero relaxation time of the fluid, as well as the nonlinearities are naturally present even in the simplest model, like upper convected Maxwell (UCM) model. Hence, unlike the Newtonian fluid, the UCM fluid flow over a rigid surface may become unstable under certain critical conditions. However, the linear stability analysis of Gorodtsov \& Leonov (1967) concluded decisively that the flow remains stable for any value of fluid elasticity, given by the Weissenberg number. As rendering the solid wall deformable is known to introduce instability, Shankar \& Kumar (2004) found that the UCM fluid flow becomes unstable when the solid surface is made deformable beyond a critical value. In this analysis, the deformable solid was modeled as Hookean elastic continuum. Under the viscous flow conditions, the strain in the solid is of the order unity, making the linear elastic model unsuitable. The most appropriate description of the elastic solid continuum is neo-Hookean elastic solid. Here, we provide a brief review of the stability analysis to examine the role of fluid elasticity on the viscous mode of instability. The details are reported in Chokshi \& Kumaran (2007a). 


\subsection{Problem formulation}

The plane shear flow of an incompressible viscoelastic fluid of density $\rho$ and viscosity $\eta$ is generated by moving a plate at $y^{*}=R$ in $x$-direction with velocity $V$ as shown in figure 1 . The fluid is supported by a deformable solid continuum with shear modulus $G$ and thickness $H R$ which is anchored with a stationary plate at $y^{*}=-H R$. Here and in what follows, the quantities with a superscript $*$ are dimensional and the ones without the superscript are dimensionless unless stated otherwise. Upon scaling the distance with $R$, time with $\eta / G$, velocity with $G R / \eta$ and stresses with $G$, the dimensionless fluid governing equations are

$$
\nabla \cdot \mathbf{v}=0, \quad \nabla \cdot \tau=0,
$$

where $\mathbf{v}$ denotes the fluid velocity field. For the Oldroyd-B viscoelastic fluid, the total stress tensor $\boldsymbol{\tau}$ is

$$
\boldsymbol{\tau}=-p_{f} \mathbf{I}+\boldsymbol{\tau}^{s}+\boldsymbol{\tau}^{p},
$$

where I is the identity tensor. The dimensionless viscous stress arising due to the solvent viscosity $\left(\eta_{s}\right)$ is of the form

$$
\boldsymbol{\tau}^{s}=\beta\left[\nabla \mathbf{v}+(\nabla \mathbf{v})^{T}\right]
$$

where the superscript $T$ indicates the transpose. The parameter $\beta=\eta_{s} / \eta$. The polymeric stress $\tau^{p}$ is described by the dimensionless Oldroyd-B constitutive model:

$$
\boldsymbol{\tau}^{p}+W \mathscr{D}_{t} \boldsymbol{\tau}^{p}=(1-\beta)\left(\nabla \mathbf{v}+(\nabla \mathbf{v})^{T}\right),
$$

where Weissenberg number $W_{i}$ is the dimensionless relaxation time of the fluid defined as $W=$ $(\lambda G / \eta)$ is the measure of the elasticity of the viscoelastic fluid. Here, $\lambda$ is dimensional relaxation time of polymer solution. The material time derivative $\mathscr{D}_{t} \tau^{p}$ is upper convected time derivative defined as

$$
\mathscr{D}_{t} \boldsymbol{\tau}^{p}=\partial_{t} \boldsymbol{\tau}^{p}+\mathbf{v} \cdot \nabla \boldsymbol{\tau}^{p}-\boldsymbol{\tau}^{p} \cdot(\nabla \mathbf{v})-(\nabla \mathbf{v})^{T} \cdot \boldsymbol{\tau}^{p} .
$$

The momentum conservation equation for the Newtonian fluid is recovered in the limit $W \rightarrow 0$ as well as for $\beta=1$ and the governing equation for the upper convected Maxwell (UCM) fluid is obtained for $\beta=0$.

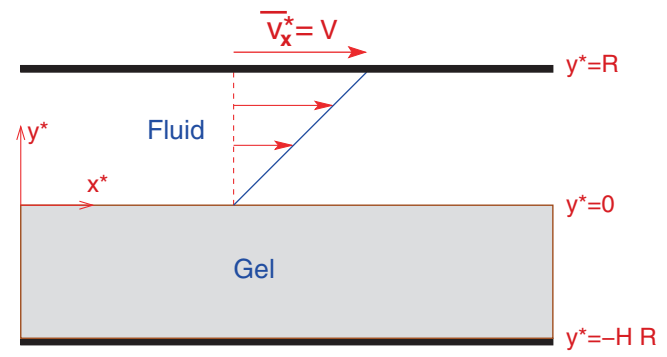

Figure 1. Schematic diagram of plane Couette flow over a flexible surface showing the dimensional coordinate system. 
The deformable wall is modeled as an incompressible neo-Hookean viscoelastic solid continuum. In Eulerian description, the dynamics of the elastic solid is described by a displacement field $\mathbf{u}$. The dimensionless momentum balance equation, in the absence of inertia, is

$$
\nabla \cdot \sigma=0
$$

The constitutive relation for the dimensionless stress, scaled with the shear modulus $G$, is

$$
\boldsymbol{\sigma}=-p_{g} \mathbf{I}+2 \mathbf{e}+\boldsymbol{\sigma}^{V}
$$

The strain tensor in the neo-Hookean elastic solid is given by (Malvern 1969):

$$
\begin{gathered}
\mathbf{e}=\frac{1}{2}\left(\mathbf{I}-\mathbf{f}^{T} \cdot \mathbf{f}\right) \quad \text { or } \\
e_{i j}=\frac{1}{2}\left(\frac{\partial u_{i}}{\partial x_{j}}+\frac{\partial u_{j}}{\partial x_{i}}-\frac{\partial u_{k}}{\partial x_{i}} \frac{\partial u_{k}}{\partial x_{j}}\right) .
\end{gathered}
$$

The viscous stress tensor $\sigma^{V}$ is taken to be zero for pure elastic solid.

The mass conservation condition for an incompressible gel is given by

$$
\nabla \cdot \mathbf{v}^{g}=0
$$

where $\mathbf{v}^{g}$ is the dimensionless Eulerian velocity field in the gel medium given by

$$
\mathbf{v}^{g}=\partial_{t} \mathbf{u}+\mathbf{v}^{g} \cdot \nabla \mathbf{u}
$$

For the steady-state base flow shown in figure 1 , the fluid velocity field is $\overline{\mathbf{v}}=(\Gamma y, 0,0)$, the solid displacement field is $\overline{\mathbf{u}}=[\Gamma(y+H), 0,0]$. The steady-state shear stress in fluid is $\bar{\tau}_{x y}=\Gamma$. By continuity, the shear stress in elastic solid is also $\bar{\sigma}_{x y}=\Gamma$. Here, $\Gamma=V \eta /(G R)$ is an important flow parameter indicating dimensionless shear rate in the fluid, and also the dimensionless strain in the elastic solid. The first normal-stress difference in solid is non-zero at $\left(\bar{\sigma}_{x x}-\bar{\sigma}_{y y}\right)=\Gamma^{2}$, which is the consequence of the neo-Hookean constitutive model which retains the terms quadratic in displacement gradient $\Gamma$. The viscoelastic fluid also exhibits the non-zero first normal-stress difference $\left(\bar{\tau}_{x x}-\bar{\tau}_{y y}\right)=2(1-\beta) W \Gamma^{2}$, which vanishes in the limit of Newtonian fluid $(\beta=1$ or $W \rightarrow 0)$.

\subsection{Linear stability analysis}

In this section, we review the linear stability analysis for the viscoelastic flow over a neoHookean elastic solid ignoring the viscous dissipation in the solid. The two-dimensional normal mode perturbations of the following form are superimposed on the base-state flow:

$$
\phi^{\prime}=\tilde{\phi}(y) e^{(i k x+s t)},
$$

where $k$ is the stream-wise wavenumber, $s$ is complex valued growth rate and $\phi$ represents the perturbation variable in fluid and solid, $\phi=\left[\mathbf{v}, p_{f}, \boldsymbol{\tau}, \mathbf{u}, p_{g}\right]$. After linearizing the governing equations using disturbance amplitude as a small parameter, and setting the linear growth rate Real $(s)$ equal to zero, a neutral stability diagram for transition shear rate $\Gamma_{t}$ is constructed. Figure 2 shows a typical neutral stability diagrams. Due to non-zero normal stress difference in 
the neo-Hookean solid, there exists a shortwave instability mode in addition to the finite wave instability of Kumaran et al (1994). As shown in figure 2, while the finite wave mode is the critical mode for thick solids with $H>1$, for thin solids with $H \leq 1$, the most unstable mode is the shortwave mode. The latter is an instability localized at the fluid-solid interface and is unaffected by the thickness of solid.

As the fluid becomes viscoelastic, the Weissenberg number $W=\lambda G / \eta$ increases from zero. The effect of Weissenberg number on $\Gamma_{c}$ is depicted in figure 3(a) for thin solids with $H=1$. Here, the instability is governed by the shortwave mode. For $\beta \geq 0.5$, which represents the dilute polymeric solutions, $\Gamma_{c}$ decreases marginally below its Newtonian value before diverging at certain Weissenberg number $W \approx 1$. Thus, the viscous instability ceases to exist for viscoelastic fluid with $W>1$, indicating strong stabilizing role of fluid elasticity for dilute solutions. However, for $\beta<0.5$, representing concentrated polymeric solution as well as polymer melt $(\beta=0)$, the instability persists even for very large value of Weissenberg number.

Similar plot for the thick solids is shown in figure 3(b). It should be noted that for this case, the instability in Newtonian fluid is of finite wave nature. Here again, for dilute solutions with $\beta \geq 0.5$, the instability is completely suppressed as $\Gamma_{c}$ diverges at a certain Weissenberg number, proportional to solid thickness $H$. For concentrated solutions and melt, i.e. with $\beta<0.5$, the
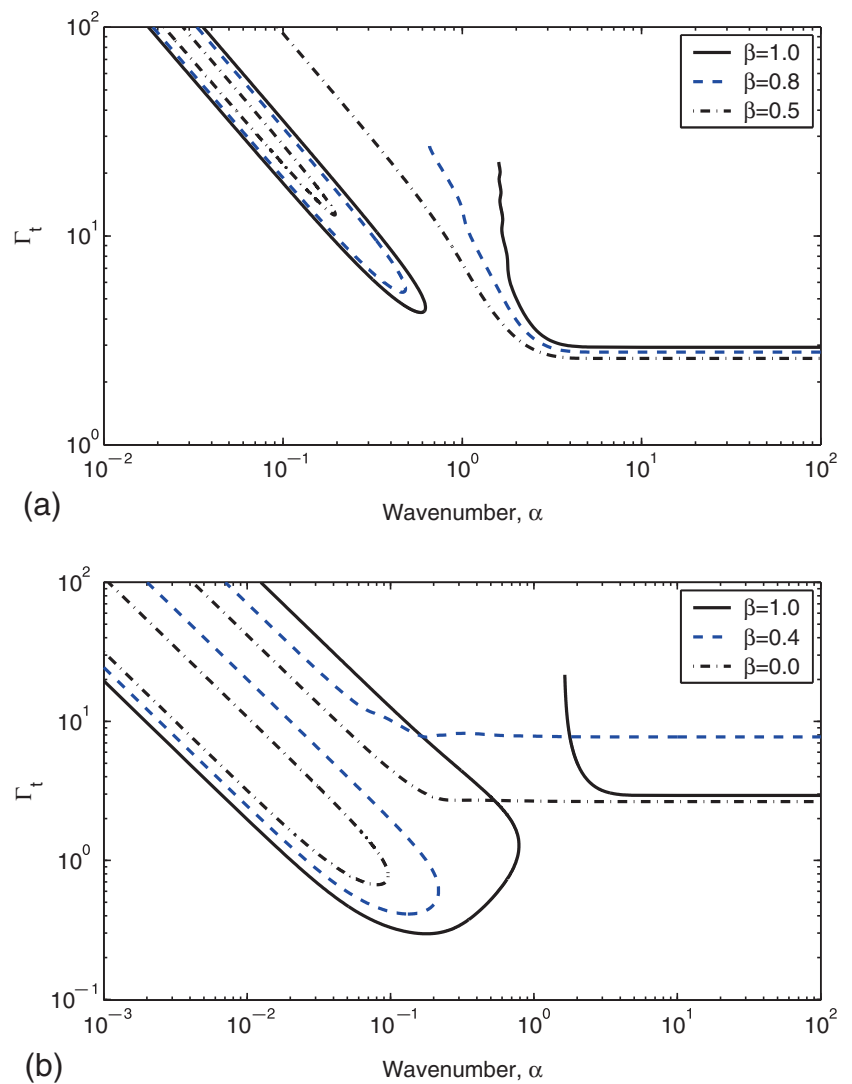

Figure 2. Neutral stability curve showing the transition shear rate $\Gamma_{t}$ as a function of wavenumber $k$. Note: the wavenumber $\alpha$ is same as the disturbance wavenumber $k$ in Eq. (12). (a) $H=1, W=0.15$; (b) $H=10, W=5$. Interfacial tension $T$ is kept zero [Source: Chokshi \& Kumaran 2007a]. 

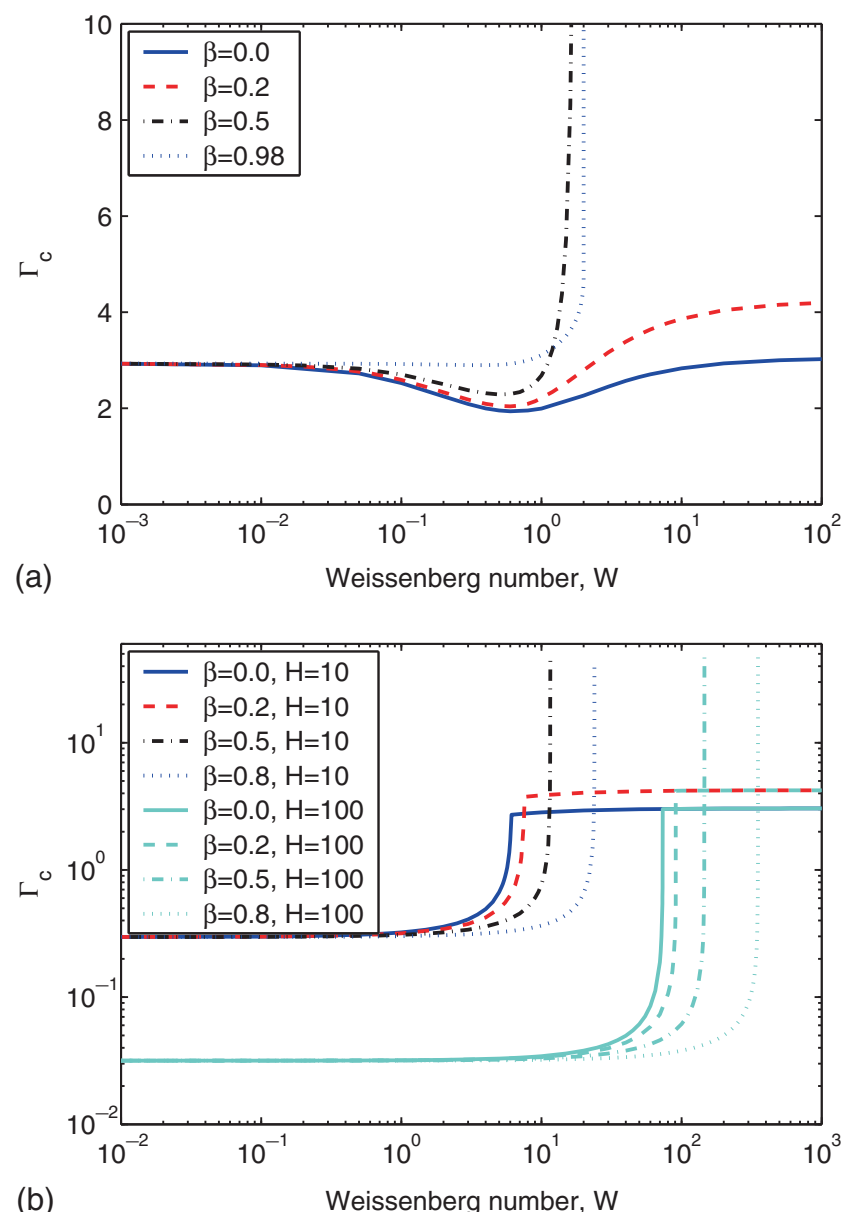

Figure 3. Variation of the critical shear rate $\Gamma_{c}$ with Weissenberg number: (a) $H=1$. The instability in thin solids is driven by the shortwave mode; (b) $H=10$ and $H=100$. The plateau in $\Gamma_{c}$ represents the highly elastic shortwave mode [Source: Chokshi \& Kumaran 2007a].

shortwave instability mode exists for entire range of Weissenberg number as shown in figure 3(a), hence the critical mode crosses over from the finite wavenumber mode (diverging branch) to the shortwave mode at certain Weissenberg number. For $\beta<0.5$, the plateau value of $\Gamma_{c}$ for high Weissenberg number indicates the shortwave instability of interface. Because of the shortwave nature, the value of $\Gamma_{c}$ is independent of solid thickness $H$.

With the focus on dilute polymeric systems ( $\beta$ close to unity), the scaling for the Weissenberg number for suppression of instability with $H$ is established in figure 4 for thick solids $(H \geq 10)$. The critical shear rates $\Gamma_{c}$ for different solid thickness $H$ collapse on to a single curve in the limit $H \gg 1$. This suggests the Weissenberg number for diverging $\Gamma_{c}$, that is complete suppression of instability, scales with solid thickness $H$.

Above results are plotted in the form of the variation of $\Gamma_{c}$ with solid thickness $H$ for different values of $W$ in figure 5. Here, $\beta=0.5$, the dilute solution limit, for which case the instability is suppressed irrespective of solid thickness $H$ for certain large value of Weissenberg number. The 




Figure 4. $\Gamma_{c} H$ as a function of the scaled Weissenberg number $W / H$ for $\beta=0.8$. The data for different values of $H$ fall on a single curve in the limit $H \gg 1$ [Source: Chokshi \& Kumaran 2007a].

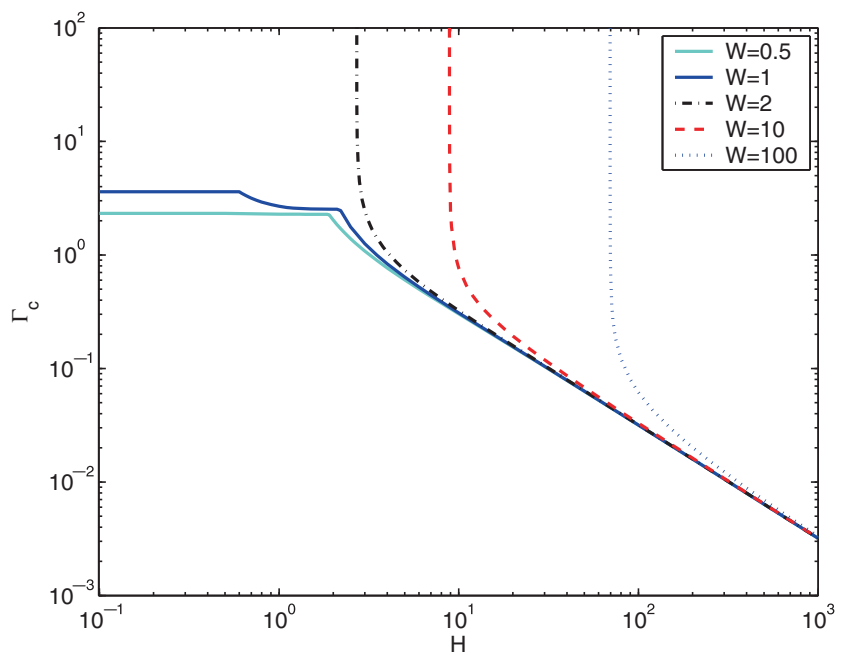

Figure 5. Effect of solid thickness $H$ on the critical shear rate $\Gamma_{c}$ for $\beta=0.5$. The plateau in $\Gamma_{c}$ for thin solids represents the shortwave instability [Source: Chokshi \& Kumaran 2007a].

plateau in $\Gamma_{c}$ for $H<1$ indicates the shortwave mode. For $H \gg 1, \Gamma_{c}$ is shown to decrease proportional to $H^{-1}$.

To outline the stability behavior for viscoelastic fluids, figure 6 shows a stability map valid for thick solids specifying the regions where the different instability modes are critical. For small Weissenberg number $(W / H<1)$, the finite wavenumber instability is present for all values of $\beta$. As mentioned earlier, $\Gamma_{c}$ for this mode increases with $W$ and diverges at $W / H \approx 1$, leading to the disappearance of finite wavenumber instability for $W \gg 1$. In the limit of high Weissenberg number, the shortwave instability is, however, present for concentrated solutions 


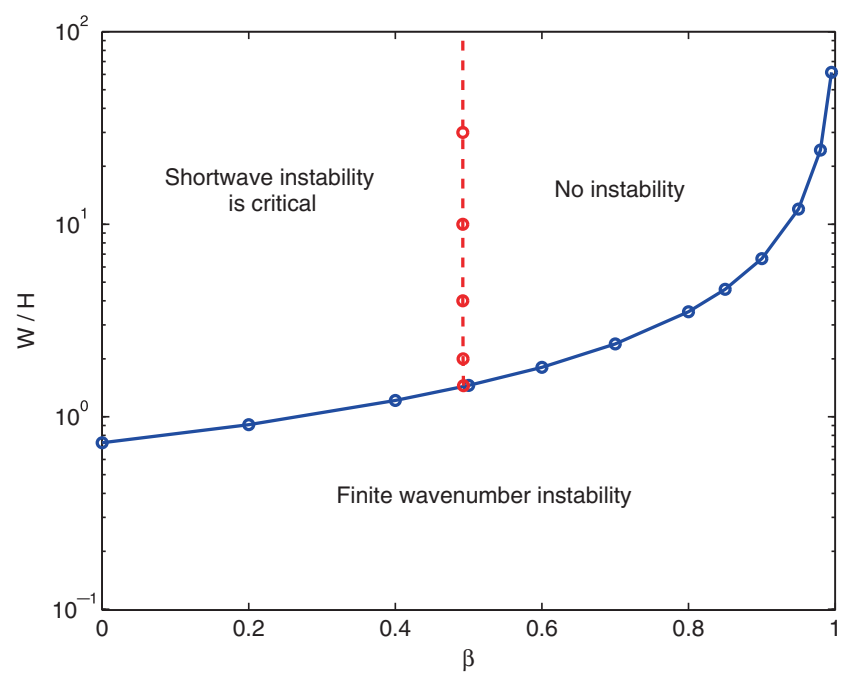

Figure 6. Stability behavior in the parametric space $W / H-\beta$. This plot holds for $H \gg 1$. For any point along the solid line, $\Gamma_{c}$ for the finite wavenumber mode diverges as $W$ is increased. And for any point along the broken line, $\Gamma_{c}$ for the shortwave mode diverges as $\beta$ is increased [Source: Chokshi \& Kumaran 2007a].

with $\beta<0.5$. Thus, we have highly elastic shortwave instability for polymer melts. However, the region $0.5 \leq \beta<1$, representing the dilute polymer solutions, witnesses no instability in the limit for sufficiently viscoelastic fluid with $W \gg 1$.

For Newtonian fluid, the interfacial tension is known to eliminate the shortwave instability (Renardy 1988; Gkanis \& Kumar 2003) arising due to neo-Hookean solid. However, the shortwave instability for highly viscoelastic fluids, as indicated by the plateau in figure 3 for $W>1$, remains unaffected by introduction of the interfacial tension at fluid-solid interface (Chokshi \& Kumaran 2007a).

\section{High Reynolds number flow}

In the limit of high Reynolds number, the disturbance vorticity is confined to a small wall layer of thickness $O\left(R e^{-1 / 3}\right)$ times the channel width. These modes are referred to as the wall modes. For Newtonian fluids flowing over an elastic solid, the wall modes are unstable (Shankar \& Kumaran 2001, 2002). Thus, for a given fluid-solid system, there exists a critical Reynolds number beyond which the flow becomes unstable to wall modes. Importantly for the Newtonian fluids, the first most unstable wall mode is, in fact, the numerical continuation of the unstable viscous mode for non-zero Reynolds number. There has been no published study of wall modes for the viscoelastic flow. Here, we present the only analysis carried out for viscoelastic wall modes (Chokshi et al 2015).

\subsection{Problem formulation}

The base flow configuration is the same as that shown in figure 1. For high Reynolds number flow, the appropriate velocity scale is $\sqrt{G / \rho}$, obtained by balancing elastic stresses in the solid with the inertial stresses in the fluid. Upon scaling distance with $R$, time with $R \sqrt{\rho / G}$ and 
stresses in fluid as well as in elastic wall with shear modulus $G$, the dimensionless governing equations are

$$
\begin{gathered}
\nabla \cdot \mathbf{v}=0, \\
D_{t} \mathbf{v}=-\nabla p^{f}+\nabla \cdot \boldsymbol{\tau},
\end{gathered}
$$

For viscoelastic fluid, the deviatoric stress tensor $\tau$ consists of viscous stress due to solvent $\boldsymbol{\tau}^{s}$ and the polymeric stress $\boldsymbol{\tau}^{p}, \boldsymbol{\tau}=\boldsymbol{\tau}^{s}+\boldsymbol{\tau}^{p}$. The viscous stresses arising due to the solvent viscosity $\eta_{s}$ are given by the Newton's law of viscosity:

$$
\boldsymbol{\tau}^{s}=\beta \frac{\Gamma_{i}}{R e}\left(\nabla \mathbf{v}+(\nabla \mathbf{v})^{T}\right)
$$

Here $\Gamma_{i}=\sqrt{\rho V^{2} / G}$ is the flow parameter, indicating the dimensionless top plate velocity. Physically, $\Gamma_{i}$ is the ratio of inertial stresses in the fluid to the elastic stresses in the solid. The parameter $\beta=\eta_{s} / \eta$ indicates the solvent contribution to the solution viscosity. The polymeric stress $\tau^{p}$ is expressed with the help of dimensionless Oldroyd-B constitutive model:

$$
\boldsymbol{\tau}^{p}+W_{i} \mathscr{D}_{t} \boldsymbol{\tau}^{p}=(1-\beta) \frac{\Gamma_{i}}{R e}\left(\nabla \mathbf{v}+(\nabla \mathbf{v})^{T}\right),
$$

where Weissenberg number $W_{i}$ is the dimensionless relaxation time of the fluid defined as $W_{i}=$ $(\lambda / R) \sqrt{G / \rho}$ is the measure of the elasticity of the viscoelastic fluid. It should be noted that the Weissenberg number for high Reynolds number analysis is different than the one in used in Section 2 for the viscous flow. The material time derivative $\mathscr{D}_{t} \tau^{p}$ is upper convected time derivative given by Eq. 5 .

For the high Reynolds number flow, the base-state strain in the solid is found to be of the order of $R e^{-1 / 3}$ for $R e \gg 1$ (Shankar \& Kumaran 2002). As the solid strain is much less than unity, one can use the Hookean elastic model to describe the dynamics in the solid medium. Hence, the Hookean model is employed to describe the incompressible linearly elastic solid. The dynamics of the elastic solid medium is described by the displacement field $\mathbf{u}$, scaled by fluid thickness $R$. The governing equations for the solid medium are

$$
\begin{gathered}
\nabla \cdot \mathbf{u}=0, \\
D_{t}^{2} \mathbf{u}=-\nabla p_{g}+\nabla \cdot \boldsymbol{\sigma} .
\end{gathered}
$$

Here, $\sigma$ the stress tensor for the wall, scaled by $G$, given by the dimensionless form of the linear elastic constitutive model:

$$
\boldsymbol{\sigma}=\left[\nabla \mathbf{u}+(\nabla \mathbf{u})^{T}\right] .
$$

For the steady-state, the velocity field is $\overline{\mathbf{v}}=\left(\Gamma_{i} y, 0,0\right)$, the solid displacement is $\bar{u}_{x}=$ $\Gamma_{i}^{2} / \operatorname{Re}(y+H)$, the shear stress is fluid and solid are $\bar{\tau}_{x y}=\bar{\sigma}_{x y}=\Gamma_{i}{ }^{2} / R e$. While normalstresses in the elastic solid are zero (for Hookean model), the Oldroyd-B viscoelastic fluid exhibits non-zero first normal-stress difference $N_{1}=\left(\bar{\tau}_{x x}-\bar{\tau}_{y y}\right)=2(1-\beta) W_{i} \Gamma_{i}^{3} / R e$.

\subsection{Linear stability analysis}

For non-zero Reynolds number flow, we have two parameters which contain the top-plate velocity $V$, viz. Re and $\Gamma$. One of these two may be considered as the critical parameter for the onset of instability. The extent of wall deformability is expressed in another dimensionless parameter $\Sigma$ defined as $\Sigma=\rho G R^{2} / \eta^{2}$, a flow independent dimensionless parameter characterizing 
the fluid-solid system. The linear stability analysis provides the critical Reynolds number for a given fluid-solid system:

$$
\operatorname{Re}_{c}=\mathscr{F}(\Sigma, \beta, W e, H) .
$$

The effect of fluid elasticity on the most unstable wall mode is depicted in figure 7 for $\Sigma=$ 5000 and $\beta=0.5$. The critical Reynolds number $R e_{c}$ in the limit $W e \rightarrow 0$ represents the most unstable mode in the class of wall modes reported by Shankar \& Kumaran (2002). Interestingly for $W e>1$, there exist multiple branches, and hence multiple critical values for Reynolds number. For critical stability, however, one is interested only in the lowest value of $R e_{c}$. For thick solids with $H=2 \& 3$ shown in this figure, $R e_{c}$ for the viscoelastic fluid is found to be much lower than its values for the Newtonian fluids, thus indicating the destabilizing role of fluid elasticity.

For practical purposes, one is interested in the critical Reynolds number for a fluid-solid system with a specific extent of wall deformability, given by the dimensionless parameter $\Sigma$. Such a neutral stability diagram for the most unstable branch of the wall mode is constructed in $R e_{c^{-}} \Sigma$ plane for different viscoelastic parameters $\beta$ and $W_{i}$ in figure 8 . The curve for $\beta=1.0$ represents the Newtonian wall mode, and is shown for comparison. The addition of polymer is captured by value of $\beta$ below unity. It is seen that for $\beta<1$, the stability curve for the polymeric fluid lies below the curve for the Newtonian fluid. Thus, the value of $\operatorname{Re}_{c}$ decreases upon addition of polymer. Thus, increasing concentration of polymer has destabilizing influence on the wall mode of instability. The reduction in critical Reynolds number is observed for a range of Weissenberg number studied. Moreover, the critical Reynolds number is found to follow the Newtonian scaling law of $R e \sim \Sigma^{3 / 4}$. This shows that the viscoelastic wall mode is qualitatively similar to the Newtonian wall mode. The analysis, therefore, shows that the critical Reynolds number can be reduced by up to one order of magnitude by introducing viscoelasticity in the fluid by polymer addition.

As discussed earlier with the help of figure 7, the viscoelasticity exhibits two kinds of unstable modes - one attributed to the lower isolated domain (which has destabilizing role of polymer)

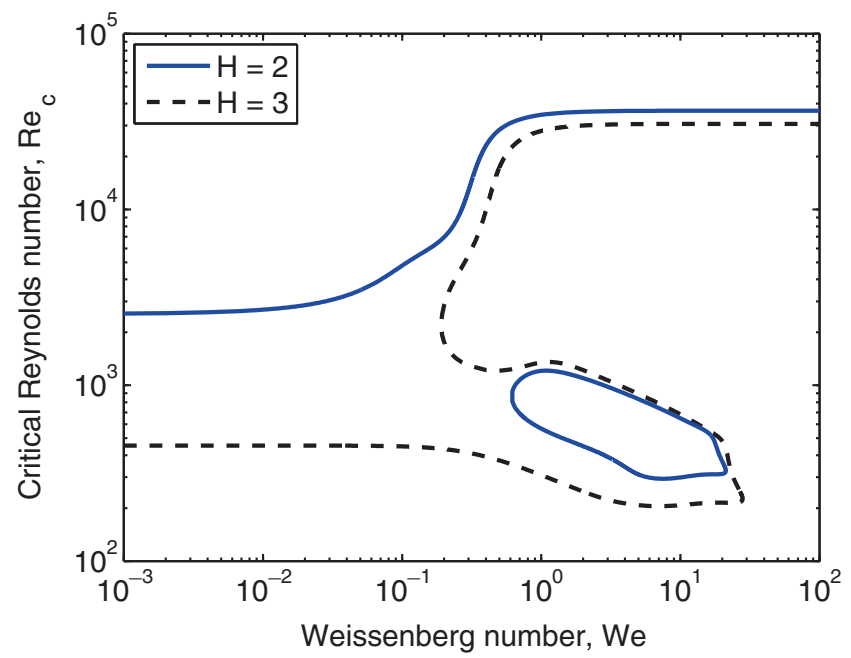

Figure 7. Effect of fluid elasticity on Newtonian wall mode: critical Reynolds number $R e_{c}$ as a function of Weissenberg number $W_{i}$ for $\Sigma=5000, \beta=0.5, k=1$ and different values of solid thickness $H$. 'S' means stable and 'U' means unstable region [Source: Chokshi et al 2015]. 


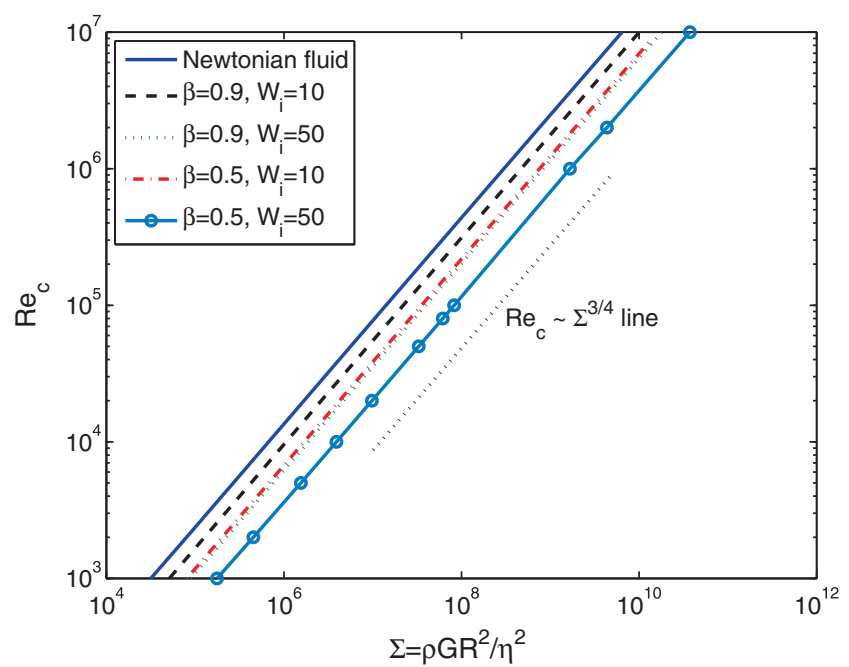

Figure 8. Stability diagram in $R e_{c}-\Sigma$ plane for $H=5$ and varying values of $\beta$ and $W_{i}$. The destabilizing role of fluid elasticity is evident. For different values of viscoelasticity parameters $R e_{c}$ scales as $\Sigma^{3 / 4}$ [Source: Chokshi et al 2015].

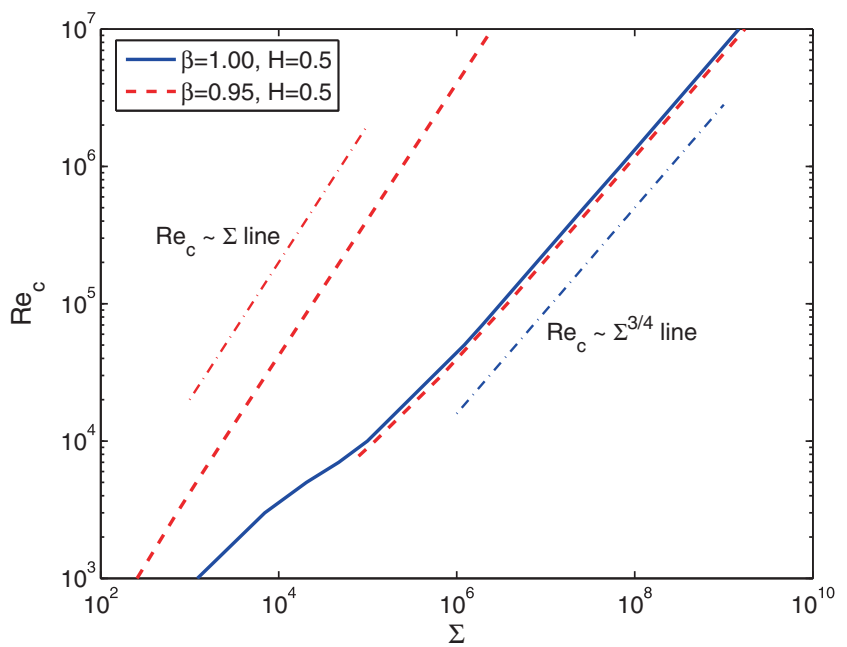

Figure 9. Neutral stability curve in $\operatorname{Re}_{c}-\Sigma$ plane for $H=0.5$. The broken lines are for $\beta=0.95$ and $W_{i}=5$ representing very dilute solution. The dual role of polymer addition is visible. The upper branch which follows $\operatorname{Re}_{c} \sim \Sigma$ exists for all Reynolds number. At $R e \approx 7760$, the lower branch appears and it is the most critical mode higher Reynolds number. For $R e \lesssim 7760$, the role of polymer is strong stabilizing [Source: Chokshi et al 2015].

and the other which shows the stabilizing effect of elasticity. The critical stability diagram in $R e_{c}-\Sigma$ plane is constructed using the most unstable mode. Therefore, in cases when the lower domain exists, the critical mode lies below the Newtonian wall mode, suggesting the destabilizing influence of viscoelasticity. To demonstrate the opposite role of viscoelasticity, figure 9 shows the stability diagram for $H=0.5$ and $\beta=0.95$ for which case the lower isolated domain 
disappears at least for low Reynolds number (around 1000). In this case, the upper structure with $\Gamma_{t}$ greater than its Newtonian value is the only mode, and hence critical. Upon continuing this mode to higher Reynolds number, the stability curve remains above the Newtonian curve. Interestingly, this mode of instability follows the scaling $R e_{c} \sim \Sigma$, a departure from the Newtonian wall mode behavior. Upon increasing $R e$, the lower isolated domain appears at $R e \approx 7760$. Hence, the most critical mode crosses over from the stabilizing one to the destabilizing one which, as shown in figure 9, lies marginally below the Newtonian curve and follows the Newtonian scaling law of $R e \sim \Sigma^{3 / 4}$. Thus, of the two curves shown for $\beta=0.95$, the bottom one, indicating the destabilizing influence of polymer addition, is the real critical stability curve for any $R e \gtrsim 7760$. It is observed that the lower isolated domain grows bigger upon increasing the Reynolds number, and it shrinks and disappears upon reducing the Reynolds number.

To summarize, for thick solids ( $H \gtrsim 1$ ), the fluid elasticity has a destabilizing effect on the Newtonian wall mode for entire range of parameter $\beta$ (all concentration levels of polymer) and the viscoelastic wall mode follows the scaling law of $R e_{c} \sim \Sigma^{3 / 4}$ for high Reynolds number. On the other hand, for thin solids $(H \lesssim 1)$, the fluid elasticity has a stabilizing influence on the Newtonian wall mode for very dilute polymer solutions $(0.8 \lesssim \beta<1)$, whereas the influence is destabilizing for $\beta \lesssim 0.8$. Here, the stabilizing influence is accompanied by scaling law of $R e_{c} \sim \Sigma$, and the destabilizing effect follows $R e_{c} \sim \Sigma^{3 / 4}$. Figure 10 shows the schematic illustration of this summary in $\beta$-H domain, plotted for $R e \approx 1100$. As mentioned before, the region in $\beta-H$ space with destabilizing influence also grows with Reynolds number. The stabilizing role of polymer addition, on the other hand, can be seen in a region of dilute polymer solution, which grows upon decreasing the Reynolds number. It should be noted that the words 'stabilizing' and 'destabilizing' mean respective increase or decrease of the critical Reynolds number by polymer addition in comparison to the Newtonian value of $R e_{c}$.

\subsection{Relationship between wall mode and viscous mode}

In this Section, we relate the wall modes analyzed in Section 3.2 for $R e \gg 1$ to the viscous mode instability of creeping flow in Section 2. The lower isolated domain for the wall mode in

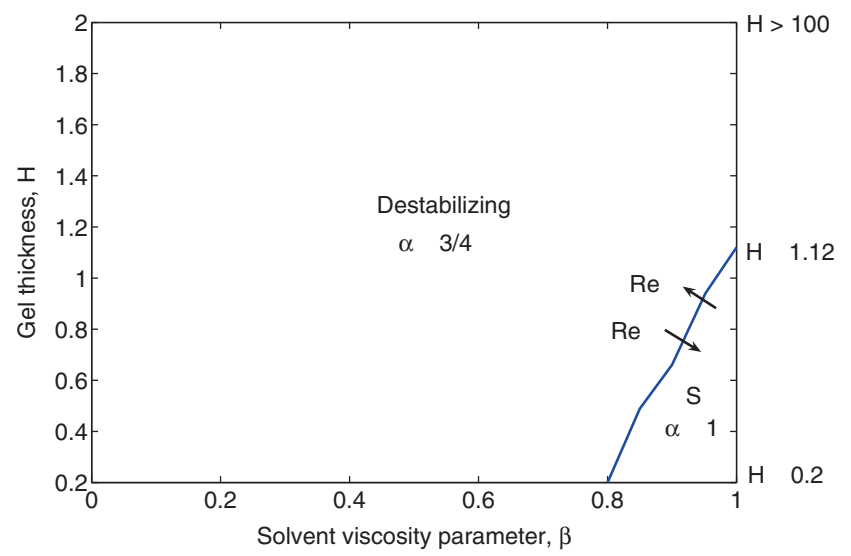

Figure 10. Schematic diagram showing qualitatively the region of stabilizing and destabilizing effects of polymer addition on the least stable Newtonian wall mode in $\beta-H$ space valid for $R e \approx 1100$ and any Weissenberg number under 100. Here, 'S' stands for stabilizing influence and $\alpha$ is the exponent in the power law scaling $R e \sim \Sigma^{\alpha}$ [Source: Chokshi et al 2015]. 
figure 7, which shows the destabilizing effect of fluid elasticity, appears to shrink upon decreasing Reynolds number, such that it is absent in the limit $R e \rightarrow 0$. Thus, for any given set of parameters, the destabilizing branch ceases to exist at a certain low value of Reynolds number, where as it always exists in the limit $R e \gg 1$. A typical case is for $H=0.5$ shown earlier in figure 9, where the lower isolated domain disappears below $R e \approx 7760$. It is observed that the thicker the solid, the lesser is the value of Reynolds number where the lower branch disappears. Note that the lower branch, whenever it exists, represents the most unstable mode with lowest $R e_{c}$. For this mode, $R e_{c} \sim \Sigma^{3 / 4}$ in the limit $R e \gg 1$ (see figure 10), and it does not continue for $R e \ll 1$. Thus, the most unstable wall mode for the viscoelastic fluid does not continue to the viscous mode. This finding is contrary to the Newtonian fluid for which case the most unstable wall mode is numerical continuation of the unstable viscous mode (Shankar \& Kumaran 2002).

\section{Conclusions}

In the present paper, we review the linear stability behavior of the flow past a deformable solid medium of finite thickness. The emphasis has been on the role of fluid elasticity in the instability modes. In particular, we revisit two kinds of instability modes: the viscous mode in the limit of $R e \rightarrow 0$, and the wall mode, in the limit $R e \gg 1$. For the creeping flow, there exist two kinds of viscous modes - the finite wavenumber mode and the shortwave mode. The latter arises due to nonlinear elastic behavior described by the neo-Hookean model for the deformable solid medium. The fluid elasticity, given by the Weissenberg number, has stabilizing influence on both classes of viscous modes. Increasing Weissenberg number, in most cases, tends to increase the critical shear rate $\Gamma_{c}$, thus delaying the onset of viscous instability. For very highly elastic fluids, the stability behavior is strongly influenced by polymer concentration parameter $\beta$. For dilute polymeric solution with $\beta>0.5$, the instability is completely suppressed for certain high value of Weissenberg number, proportional to solid thickness $H$. On the other hand, for highly concentrated solutions and polymer melt with $\beta<0.5$, the shortwave instability exists, and hence the highly elastic fluids exhibit instability with $\Gamma_{c}$ larger than its value for the Newtonian fluid.

For very high Reynolds number flow, the critical Reynolds number for transition is significantly lower for the viscoelastic fluid than its value for the Newtonian fluid. Thus, the fluid elasticity shows destabilizing influence on the Newtonian wall mode. Moreover, the critical Reynolds number scales with $\Sigma=\rho G R^{2} / \eta^{2}$, the parameter characterizing the fluid-solid system, as $R e \sim \Sigma^{3 / 4}$ for $R e \gg 1$, the same as the Newtonian wall mode. This scaling holds for all values of $\beta$ and $W_{i}$. However, for a moderate Reynolds number in the range of $10^{3}-10^{4}$, the role of fluid elasticity can be either destabilizing or stabilizing in comparison to the Newtonian fluid depending upon the polymer concentration parameter $\beta$. For very dilute polymer solutions with $\beta=\eta_{s} / \eta$ just under unity $(0.9 \lesssim \beta<1)$ and for thin solids with $H \lesssim 1.1$, the above-mentioned branch with scaling $\operatorname{Re}_{c} \sim \Sigma^{3 / 4}$ (destabilizing role of polymer) disappears and the most unstable branch follows the scaling $R e_{c} \sim \Sigma$, a departure from the Newtonian wall mode behavior. This implies that the critical Reynolds number in this regime is higher for the viscoelastic fluid than its value for the Newtonian fluid. Thus, very small addition of polymer molecules tends to delay the transition indicating stabilizing role of fluid elasticity.

Except for a narrow region of parameters in $\beta-H$ space, increasing fluid elasticity renders the wall modes more unstable. This destabilizing role of polymer at high Reynolds number wall modes is contrary to the stabilizing effect of elasticity in for the viscous mode in the absence of inertia. It has been shown that the most unstable viscoelastic wall mode at high Reynolds number 
ceases to exist on decreasing the Reynolds number, thus it does not continue to the viscous mode. This behavior is different from the Newtonian case, where the most unstable wall mode is numerical continuation of the viscous mode.

\section{Acknowledgements}

Author would like to thank Piyush Bhade for making useful contributions in the analysis for high Reynolds number flow.

\section{References}

Chokshi P and Kumaran V 2007a Finite amplitude stability of viscoelastic flow past a deformable wall in the creeping flow limit. Phys. Fluids 19: 104103

Chokshi P and Kumaran V 2007b Stability of the viscous flow of a polymeric fluid past a flexible surface. Phys. Fluids 19: 034102

Chokshi P, Bhade P and Kumaran V 2015 Wall-mode instability in plane shear flow of viscoelastic fluid over a deformable solid. Phys. Rev. E 91: 023007

Corcos G M and Sellars J R 1959 On the stability of fully developed pipe flow. J. Fluid Mech. 5: 97-112

Eggert M D and Kumar S 2004 Observations of instability, hysteresis, and oscillation in low-Reynolds number flow past polymer gels. J. Colloid Interface Sci. 278: 234-242

Gill A E 1965a A mechanism for instability of plane Couette flow and of Poiseuille flow in a pipe. J. Fluid Mech. 21: 503-511

Gill A E 1965b On the behavior of small disturbances to Poiseuille flow in a circular pipe. J. Fluid Mech. 21: $145-172$

Gkanis V and Kumar S 2003 Instability of creeping Couette flow past a neo-Hookean solid. Phys. Fluids 15: $2864-2871$

Gorodtsov V A and Leonov A I 1967 On a linear instability of a plane parallel couette flow of viscoelastic fluid. J. Appl. Math. Mech. 31: 310-319

Kramer M O 1957 Boundary-layer stabilisation by distributed damping. J. Aero Sci. 24: 458-460

Krindel P and Silberberg A 1979 Flow through gel-walled tubes. J. Colloid Interface Sci. 71: 34-50

Kumar S and Shankar V 2005 Instability of high-frequency modes in viscoelastic plane Couette flow past a deformable wall at low and finite Reynolds number. J. Non-Newtonian Fluid Mech. 125: 121-141

Kumaran V 1995 Stability of the viscous flow of a fluid through a flexible tube. J. Fluid Mech. 294: 259281

Kumaran V 1996 Stability of an inviscid flow through a flexible tube. J. Fluid Mech. 320: 1-17

Kumaran V 1998a Stability of fluid flow through a flexible tube at intermediate Reynolds number. J. Fluid Mech. 357: 123-140

Kumaran V 1998b Stability of wall modes in a flexible tube. J. Fluid Mech. 362: 1-15

Kumaran V 2000 Classification of instabilities in the flow past flexible surfaces. Current Sci. 79: 776-773

Kumaran V and Muralikrishnan R 2000 Spontaneous growth of fluctuations in the viscous flow of a fluid past a soft interface. Phys. Rev. Lett. 84: 3310-3313

Kumaran V, Fredrickson G H and Pincus P 1994 Flow induced instability of the interface between a fluid and a gel at low Reynolds number. J. Phys. II 4: 893-904

Malvern L E 1969 Introduction to the mechanics of a continuous medium. Englewood Cliffs, NJ: PrenticeHall

Muralikrishnan R and Kumaran V 2002 Experimental study of the instability of the viscous flow past a flexible surface. Phys. Fluids 14: 775-780

Renardy Y 1988 Stability of the interface in two-layer Couette flow of upper convected Maxwell liquids. $J$. Non-Newtonian Fluid Mech. 28: 99-115

Renardy M 1992 Rigorous stability proof for plane couette flow of an upper convected Maxwell fluid at zero reynolds number. Eur. J. Mech. B 11: 511-516 
Shankar V and Kumar S 2004 Instability of viscoelastic plane Couette flow past a deformable wall. $J$. Non-Newtonian Fluid Mech. 116: 371-393

Shankar V and Kumaran V 2001 Asymptotic analysis of wall modes in a flexible tube revisited. Eur. Phys. J. B 19: 607-622

Shankar V and Kumaran V 2002 Stability of wall modes in fluid flow past a flexible surface. Phys. Fluids 14: $2324-2338$

Verma M K S and Kumaran V 2012 A dynamical instability due to fluid-wall coupling lowers the transition Reynolds number in the flow through a flexible tube. J. Fluid Mech. 705: 322-347

Verma M K S and Kumaran V 2013 A multifold reduction in the transition Reynolds number, and ultra-fast mixing, in a micro-channel due to a dynamical instability induced by a soft wall. J. Fluid Mech. 727: 407-455

Wilson H J, Renardy M and Renardy Y 1999 Structure of the spectrum in zero reynolds number shear flow of the UCM and oldroyd-b liquids. J. Non-Newtonian Fluid Mech. 80: 251-268 